\title{
SIMPLE GERMS OF CORANK ONE AFFINE DISTRIBUTIONS
}

\author{
MICHAIL ZHITOMIRSKII \\ Department of Mathematics, Technion \\ 32000 Haifa, Israel \\ E-mail:mzhi@techunix.technion.ac.il \\ WITOLD RESPONDEK \\ INSA de Rouen, LMI, UPRES-A CNRS 6085 \\ Place Emile Blondel, 76131 Mont Saint Aignan, France \\ E-mail: wresp@lmi.insa-rouen.fr
}

\section{Introduction}

1.1. Affine distributions. All objects are assumed to be smooth (of class $C^{\infty}$ ). An affine distribution on $R^{n}$ of rank $m$ (or corank $n-m$ ) is a family $A=\left\{A_{p}\right\}_{p \in R^{n}}$ of $m$-dimensional affine subspaces $A_{p} \subset T_{p} R^{n}$. If $A_{p}$ is a subspace, i.e., $A_{p}$ contains the zero tangent vector, then $p$ is called an equilibrium point of $A$. Two germs $A$ and $\tilde{A}$ of corank one affine distributions, at points $p$ and $\tilde{p}$ respectively, are equivalent if there exists a local diffeomorphism $\Phi$ sending $p$ to $\tilde{p}$ such that $\Phi_{*}\left(A_{x}\right)=\tilde{A}_{\Phi(x)}$ for each $x$ close to $p$.

1.2. Simple germs. Our purpose is to list simple (of zero modality) germs of affine distributions. The definition of simplicity is the same as in any local classification problem (see [AVG, 85]). Namely, a germ $A$ at a point $p$ is called simple if there exist a finite number $l$ such that $A$ is $l$-determined (which means that $A$ is equivalent to any germ $\tilde{A}$ at $p$ such that $j_{p}^{l} \tilde{A}=j_{p}^{l} A$ ) and a finite tuple of germs at the origin such that any germ at $p$ with the $l$-jet sufficiently close to $j_{p}^{l} A$ is equivalent to one of the germs of this tuple. To define $j_{p}^{l} A$ one can describe $A$ by $m+1$ vector fields $v_{0}, \ldots, v_{m}$ such that $A_{x}=v_{0}(x)+\operatorname{span}\left(v_{1}(x), \ldots, v_{m}(x)\right)$ for each $x$ close to $p$. Then $j_{p}^{l} A$ is equal (resp. close)

Research of the first author supported by the fund for promotion of research at the Technion. The second author is on leave from the Institute of Mathematics, Polish Academy of Sciences, his research was partially supported by KBN grant 2P03A 00409.

1991 Mathematics Subject Classification: Primary 58A30; Secondary 93B10.

Received by the editors: March 28, 1997; in the revised form: July 29, 1997.

The paper is in final form and no version of it will be published elsewhere. 
to $j_{p}^{l} \tilde{A}$ if the germs $A$ and $\tilde{A}$ can be described by tuples of vector fields with equal (resp. close) $l$-jets at $p$.

1.3. Why of corank one? In this paper we study corank one affine distributions only since simple dimensional arguments show that if $m \leq n-2$ then there are no simple germs, see [T, 89] and [J, 90].

1.4. Affine distributions and control systems. Any result of this paper can be reformulated in terms of control theory: the problem of local classification of affine distributions coincides with the problem of feedback classification of control affine systems, see [J, 90]. A control affine system has the form $\dot{x}=v_{0}(x)+u_{1} v_{1}(x)+\cdots+u_{m} v_{m}(x)$, where $v_{i}$ 's are vector fields ( $v_{0}$ is called a drift vector field) and $u_{i}$ 's are controls. The above given description of affine distributions via tuples of vector fields allows to pass from control affine systems to affine distributions and vice versa provided that $v_{1}, \ldots, v_{m}$ are pointwise independent. In this case two control affine systems are called feedback equivalent if they define equivalent (i.e., the same up to a choice of coordinates) affine distributions.

1.5. Germs at a nonequilibrium point: reduction to a classical problem. The starting point for classification of corank one affine distributions is the following simple observation which was made in [Zh, 92], App. C: the problem of local classification of corank one affine distributions (and the problem of local feedback classification of control affine systems on $R^{n}$ with $n-1$ controls) is exactly the classical problem of classification of nonvanishing differential 1-forms provided one studies germs at a nonequilibrium point.

To explain this observation it suffices to note that if $p$ is a nonequilibrium point of a corank one affine distribution $A$ then near $p$ there exists a unique differential 1-form $\omega$ such that

$$
A_{x}=\left\{\xi \in T_{x} R^{n}: \omega(x)(\xi)=1\right\} .
$$

Notation. This relation between $\omega$ and $A$ will be denoted by $A=(\omega, 1)$.

1.6. Simple germs at a nonequilibrium point. It was proved in [Zh, 92], Sect. 12 that any simple germ of a differential 1-form on $R^{2 k+1}$ (resp. $R^{2 k}$ ) is equivalent to one and only one of three models (fixed germs at the origin): the Darboux model $D$ (resp. $D^{\prime}$ ) and two Martinet models $M_{ \pm}\left(\right.$resp. $\left.M_{ \pm}^{\prime}\right)\left({ }^{1}\right)$, where

$$
\begin{gathered}
D=d z+x_{1} d y_{1}+\cdots+x_{k} d y_{k}, \\
M_{ \pm}= \pm z d z+\left(1+x_{1}\right) d y_{1}+x_{2} d y_{2}+\cdots+x_{k} d y_{k}, \\
D^{\prime}=\left(1+x_{1}\right) d y_{1}+x_{2} d y_{2}+\cdots+x_{k} d y_{k} \\
M_{ \pm}^{\prime}=\left(1 \pm x_{1}^{2}\right) d y_{1}+x_{2} d y_{2}+\cdots+x_{k} d y_{k} .
\end{gathered}
$$

Therefore the following statement holds:

Theorem 1 ([Zh, 92], App. C). All simple germs of corank one affine distributions at a nonequilibrium point are exhausted, up to equivalence, by the models $(D, 1)$ and $\left(M_{ \pm}, 1\right)$

$\left({ }^{1}\right)$ This result was announced in [Zh, 85] (with the proof for the 2-dimensional case). 
if $n=2 k+1$ and by the models $\left(D^{\prime}, 1\right)$ and $\left(M_{ \pm}^{\prime}, 1\right)$ if $n=2 k .\left(^{2}\right)$

Using results of the works [M, 70] and [Zh, 92], Sect. 11, one can easily determine whether a germ $(\omega, 1)$ is simple or not. Namely, given a germ $A=(\omega, 1)$ at a point $p \in R^{2 k+1}$ (resp. $R^{2 k}$ ) we take a nondegenerate volume form $\Omega$ and define the function $F=\left(\omega \wedge(d \omega)^{k}\right) / \Omega$ (resp. $F=(d \omega)^{k} / \Omega$ ). Then $A$ is equivalent to the Darboux model if and only if $F(p) \neq 0$ and to one of the Martinet models if and only if $F(p)=0$ and the $n$-form $\Omega_{1}=d F \wedge(d \omega)^{k}$ (resp. $\Omega_{1}=d F \wedge \omega \wedge(d \omega)^{k-1}$ ) does not vanish at $p$. For any $n$ the sign + (resp. - ) in the Martinet models corresponds to the case where the $n$-forms $\Omega(p)$ and $\Omega_{1}(p)$ define the same (resp. different) orientations.

1.7. Germs at an equilibrium point. Given a corank one distribution $A$ and any point $p$ (equilibrium or not) there exist a nonvanishing differential 1-form $\omega$ and a function $f$ such that for each $x$ close to $p$ we have

$$
A_{x}=\left\{\xi \in T_{x} R^{n}: \omega(x)(\xi)=f(x)\right\} .
$$

Notation. We will write this relation in the form $A=(\omega, f)$.

Note that $(\omega, f)$ and $(\tilde{\omega}, \tilde{f})$ is the same affine distribution if and only if there exists a function $T$ such that $T(p) \neq 0$ and $\tilde{\omega}=T \omega, \tilde{f}=T f$. The point $p$ is an equilibrium point of $A$ if and only if $f(p)=0$. Therefore classification of germs of corank one affine distributions at an equilibrium point $p$ is the local classification of pairs $(\omega, f), \omega(p) \neq 0$, $f(p)=0$, with respect to the following equivalence: $(\omega, f)$ is equivalent to $(\tilde{\omega}, \tilde{f})$ if there are a local diffeomorphism $\Phi$ and a nonvanishing function $T$ such that $\Phi^{*} \omega=T \tilde{\omega}, f \circ \Phi=T \tilde{f}$.

1.8. 2-dimensional and 3-dimensional cases. A classification of simple germs of corank one affine distributions on $R^{n}, n=2,3$, at an equilibrium point is contained in [JR, 90] for the 2-dimensional case and in [RZh, 95] for the 3-dimensional case (in both papers - in terms of control theory). Using the notation in Section 1.7 we can reformulate this classification as follows: any simple germ of a corank one distribution on $R^{2}$ (resp. $R^{3}$ ) at an equilibrium point is equivalent to the germ at the origin of the affine distribution $(d x, y)$ (resp. to the germ at the origin of one of the three affine distributions $(d z+x d y, y)$, $\left.\left(d z+x^{2} d y, y\right),\left(d z-x^{2} d y, y\right)\right)$.

2. New results. The new result of this paper is a complete classification of simple germs at an equilibrium point of corank one affine distributions on $R^{n}, n \geq 4$.

2.1. Classification of simple germs. In the following theorem (which is valid for $n \geq 3$ ) one meets the Darboux and Martinet models $D$ and $M_{ \pm}$in the odd-dimensional case (see Section 1.6), and the differential 1-form

$$
Q=d y_{1}+x_{2} d y_{2}+\cdots+x_{k} d y_{k}
$$

on $R^{2 k}$. Note that by one of Darboux theorems the field of kernels of $Q$ is equivalent to generic germ of corank one distribution on $R^{2 k}$. We use the notation of Section 1.7.

$\left({ }^{2}\right)$ In the 2-dimensional case $(k=1)$ this result is contained, in terms of control theory, in the work [JR, 90], where Darboux and Martinet models and results of the work [Zh, 85] are not used. 
THEOREM 2. Any simple germ at an equilibrium point of a corank one affine distribution on $R^{2 k+1}, k \geq 1$ (resp. $R^{2 k}, k \geq 2$ ), is equivalent to the germ at the origin of one and only one of the three distributions $\left(D, x_{1}\right),\left(M_{ \pm}, x_{1}\right)$ (resp. two distributions $\left(Q, x_{1}\right)$, $\left.\left(Q, x_{2}+x_{1}^{2}\right)\right)$.

2.2. Singularity classes. Now we give a way to determine whether a given germ $A=(\omega, f)$ at an equilibrium point $p$ is simple or not and, if it is, to which of the models it is equivalent. There is no loss of generality to assume that $p$ is the origin. Fix a nondegenerate volume form $\Omega$. Given a germ $A=(\omega, f)$ we define the function

$$
F=\left(\omega \wedge(d \omega)^{k}\right) / \Omega
$$

if $n=2 k+1$, and the function

$$
G=\left(\omega \wedge(d \omega)^{k-1} \wedge d f\right) / \Omega,
$$

if $n=2 k$. We introduce the following sets of germs:

the set $\operatorname{Orb}\left(D, x_{1}\right)$ consisting of germs of affine distributions $(\omega, f)$ at $0 \in R^{2 k+1}$ such that $f(0)=0, F(0) \neq 0,(\omega \wedge d f)(0) \neq 0$;

the set $\operatorname{Orb}\left(M_{+}, x_{1}\right)$ (resp. $\operatorname{Orb}\left(M_{-}, x_{1}\right)$ ) consisting of germs of affine distributions $(\omega, f)$ at $0 \in R^{2 k+1}$ such that $f(0)=0, F(0)=0$, the $n$-form $\Omega_{1}=\omega \wedge(d \omega)^{k-1} \wedge d F \wedge d f$ does not vanish at 0 and the $n$-forms $\Omega(0)$ and $\Omega_{1}(0)$ define the same (resp. different) orientations;

the set $\operatorname{Orb}\left(Q, x_{1}\right)$ consisting of germs of affine distributions $(\omega, f)$ at $0 \in R^{2 k}$ such that $f(0)=0$ and $G(0) \neq 0$;

the set $\operatorname{Orb}\left(Q, x_{2}+x_{1}^{2}\right)$ consisting of germs of affine distributions $(\omega, f)$ at $0 \in R^{2 k}$ such that $f(0)=0, G(0)=0,\left(\omega \wedge(d \omega)^{k-1} \wedge d G\right)(0) \neq 0$ and $(\omega \wedge d f)(0) \neq 0$.

One can easily check that these sets do not depend on the volume form $\Omega$ and they are well-defined disjoint sets of germs of corank one distributions: any of the above given sets is distinguished by a condition which is invariant with respect to the change of a pair $(\omega, f)$ by a pair $(T \omega, T f)$, where $T$ is a nonvanishing function.

Theorem 3. Denote by $\alpha$ any of 5 models in Theorem 2. A germ $(\omega, f)$ of a corank one affine distribution at equilibrium point $0 \in R^{n}$ is equivalent to the model $\alpha$ if and only if it belongs to the above described set $\operatorname{Orb}(\alpha)$.

In other words, $\operatorname{Orb}(\alpha)$ is the orbit of the model $\alpha$. Taking into account results of Section 1.6, we obtain that the set of all simple germs at the origin of corank one affine distributions on $R^{2 k+1}$ (resp. $R^{2 k}, k \geq 2$ ) consists of one open orbit, three codimension one orbits and two (resp. one) codimension two orbits. The set of non-simple germs has codimension two.

\section{Proofs.}

3.1. Homotopy method. Beginning from this section all objects are assumed to be germs at the origin. To prove Theorem 3 we use the homotopy method. In the following proposition all families depend smoothly on a parameter $t \in[0,1]$. By $L_{X} \omega$ we denote the Lie derivative of a differential 1-form $\omega$ along a vector field $\left.\left.X: L_{X} \omega=X\right\rfloor d \omega+d(X\rfloor \omega\right)$. 
Proposition 1. Consider a differential 1-form $\omega$, functions $f$ and $\delta$, and let $f_{t}=$ $f+t \delta$. Assume that there exist a family of vector fields $X_{t}, X_{t}(0)=0$, and a family of functions $h_{t}$, such that $L_{X_{t}} \omega=h_{t} \omega$ and $X_{t}\left(f_{t}\right)=h_{t} f_{t}-\delta$ for each $t \in[0,1]$. Then the affine distribution $(\omega, f)$ is equivalent to the affine distribution $(\omega, f+\delta)$.

Pro of. Define a family of local diffeomorphisms $\phi_{t}$ by the equation $d \phi_{t} / d t=X_{t}\left(\phi_{t}\right)$, $\phi_{0}=\mathrm{id}$, and a family of nonvanishing functions $T_{t}$ by the equation $d T_{t} / d t=\left(h_{t} \circ \phi_{t}\right) T_{t}$, $T_{0} \equiv 1$. Then

$$
\frac{d}{d t}\left(\phi_{t}^{*} \omega\right)=\phi_{t}^{*}\left(L_{X_{t}} \omega\right)=\left(h_{t} \circ \phi_{t}\right) \phi_{t}^{*} \omega, \quad \frac{d}{d t}\left(f_{t}\left(\phi_{t}\right)\right)=\left(X_{t}\left(f_{t}\right)+\delta\right) \circ \phi_{t}=\left(h_{t} f_{t}\right) \circ \phi_{t} .
$$

It follows that $\phi_{t}^{*} \omega=T_{t} \omega$ and $f_{t} \circ \phi_{t}=T_{t} f_{0}$. In particular, as $t=1$ we obtain $\phi_{1}^{*} \omega=T_{1} \omega$, $(f+\delta) \circ \phi_{1}=T_{1} f$.

3.2. Proof of Theorem 3. For each model $\alpha$ in Theorem 2 it is easy to check that $\alpha \in \operatorname{Orb}(\alpha)$. Thus it is enough to prove that if $(\omega, f) \in \operatorname{Orb}(\alpha)$ then $(\omega, f)$ is equivalent to $\alpha$.

a) Let $(\omega, f) \in \operatorname{Orb}\left(D, x_{1}\right)$. Then $\omega$ is a contact form and we can assume that $\omega=D$. The condition $(D \wedge d f)(0) \neq 0$ implies that there is no loss of generality to assume $\left(\partial f / \partial x_{1}\right)(0) \neq 0$ and, moreover, $\left(\partial f / \partial x_{1}\right)(0)>0$ (we change, if necessary, the signs of the coordinates $x_{1}$ and $y_{1}$ ). Now we will reduce $f$ to $x_{1}$ using Proposition 1 . Let $\delta=f-x_{1}$, $f_{t}=x_{1}+t \delta$. The set of solutions $(X, h)$ of the equation $L_{X} D=h D, X(0)=0$ is well known, see [Ar, 78]:

$$
\begin{gathered}
h=\frac{\partial u}{\partial z}, X=\sum A_{i} \frac{\partial}{\partial x_{i}}+\sum B_{i} \frac{\partial}{\partial y_{i}}+C \frac{\partial}{\partial z}, \\
A_{i}=x_{i} h-\frac{\partial u}{\partial y_{i}}, B_{i}=\frac{\partial u}{\partial x_{i}}, C=u-x_{1} \frac{\partial}{\partial x_{1}}-\ldots-x_{k} \frac{\partial}{\partial x_{k}},
\end{gathered}
$$

where $u$ is an arbitrary function in $n$ variables such that $\left(\partial u / \partial x_{i}\right)(0)=\left(\partial u / \partial y_{i}\right)(0)=0$. The equation $X_{t}\left(f_{t}\right)=h_{t} f_{t}-\delta$ with $X_{t}$ and $h_{t}$ of the above form for each $t$ reduces to the equation

$$
Z_{t}\left(u_{t}\right)+a_{t} u_{t}+\delta=0
$$

where $Z_{t}$ is a family of vector fields and $a_{t}$ is a family of functions. This equation has a solution $u_{t}$ with vanishing at 0 derivatives (for all $t$ ) since $\delta(0)=0$ and $Z_{t}(0) \neq 0$ for all $t \in[0,1]$. The latter follows from the relations

$$
Z_{t}\left(y_{1}\right)=-\frac{\partial f_{t}}{\partial x_{1}}, \frac{\partial f}{\partial x_{1}}(0)>0
$$

which imply $Z_{t}\left(y_{1}\right)(0)<0, t \in[0,1]$.

b) Let $(\omega, f) \in \operatorname{Orb}\left(M_{+}, x_{1}\right) \cup \operatorname{Orb}\left(M_{-}, x_{1}\right)$. Then $\left(\omega \wedge(d \omega)^{k-1} \wedge d F\right)(0) \neq 0$, therefore the field of kernels of $\omega$ is equivalent to the field of kernels of the 1-form $M_{+}$, see [M, 70]. This means that there is no loss of generality to assume that $\omega=M_{+}$. The condition $\left(M_{+}, f\right) \in \operatorname{Orb}\left(M_{+}, x_{1}\right)$ (resp. $\left.\left(M_{+}, f\right) \in \operatorname{Orb}\left(M_{-}, x_{1}\right)\right)$ is equivalent to the condition $\left(\partial f / \partial x_{1}\right)(0)>0\left(\right.$ resp. $\left.\left(\partial f / \partial x_{1}\right)(0)<0\right)$. Note that the affine distributions $\left(M_{+},-x_{1}\right)$ and $\left(M_{-}, x_{1}\right)$ are equivalent (the form $\left(-M_{+}\right)$can be obtained from $M_{-}$by the change of signs of the coordinates $\left.y_{1}, \ldots, y_{k}\right)$. Therefore to prove that $\left(M_{+}, f\right)$ is equivalent to 
$\left(M_{+}, x_{1}\right)$ (resp. to $\left.\left(M_{-}, x_{1}\right)\right)$ we have to reduce $f$ to $x_{1}$ (resp. to $\left.-x_{1}\right)$. In what follows we consider only the case $\left(M_{+}, f\right) \in \operatorname{Orb}\left(M_{+}, x_{1}\right)$. In the case $\left(M_{+}, f\right) \in \operatorname{Orb}\left(M_{-}, x_{1}\right)$ the arguments are similar.

Let $\delta=f-x_{1}, f_{t}=x_{1}+t \delta$. By Proposition 1 in order to reduce $f$ to $x_{1}$ it suffices to prove the solvability of the system $L_{X_{t}} M_{+}=h_{t} M_{+}, X_{t}\left(f_{t}\right)=h_{t} f_{t}-\delta$ with respect to a family $\left(X_{t}, h_{t}\right)$ such that $X_{t}(0)=0$. The equation $L_{X_{t}} M_{+}=h_{t} M_{+}, X_{t}(0)=0$ can be easily solved (see [RZh, 95]). The set of all solutions $\left(X_{t}, h_{t}\right)$ contains solutions of the form

$$
\begin{gathered}
h_{t}=2 v_{t}+z \frac{\partial v_{t}}{\partial z}, X_{t}=\sum A_{i, t} \frac{\partial}{\partial x_{i}}+\sum B_{i, t} \frac{\partial}{\partial y_{i}}+C_{t} \frac{\partial}{\partial z} \\
A_{1, t}=-z^{2} \frac{\partial v_{t}}{\partial y_{1}}+\left(1+x_{1}\right) h_{t}, A_{i, t}=-z^{2} \frac{\partial v_{t}}{\partial y_{i}}+x_{i} h_{t}(i=2, \ldots, k), \\
B_{i, t}=z^{2} \frac{\partial v_{t}}{\partial x_{i}}, C=z v_{t}-\left(1+x_{1}\right) z \frac{\partial v}{\partial x_{1}}-x_{2} z \frac{\partial v_{t}}{\partial x_{2}}-\cdots-x_{k} z \frac{\partial v_{t}}{\partial x_{k}},
\end{gathered}
$$

where $v_{t}$ is an arbitrary family of function in $n$ variables such that $v_{t}(0)=0$. The equation $X_{t}\left(f_{t}\right)=h_{t} f_{t}-\delta$ with such $X_{t}$ and $h_{t}$ can be written as a system for $h_{t}$ and $v_{t}$ of the form

$$
W_{t} h_{t}+z \frac{\partial f_{t}}{\partial z} v_{t}+z R_{t}\left(v_{t}\right)+\delta=0, \quad h_{t}=2 v_{t}+z \frac{\partial v_{t}}{\partial z}
$$

where $R_{t}$ is a family of vector fields such that $R_{t}(z) \equiv 0$ and $W_{t}$ is a family of functions such that $W_{t}(0)=\left(\partial f_{t} / \partial x_{1}\right)(0)$. Now we use our condition $\left(\partial f / \partial x_{1}\right)(0)>0$. It follows that $\left(\partial f_{t} / \partial x_{1}\right)(0)>0, t \in[0,1]$, and eliminating $h_{t}$ from the first equation we reduce the system to an equation for $v_{t}$ of the form

$$
\left(2+g_{t}\right) v_{t}+z \frac{\partial v_{t}}{\partial z}+z E_{t}\left(v_{t}\right)=\delta_{t},
$$

where $E_{t}$ is a family of vector fields such that $E_{t}(z) \equiv 0, g_{t}$ and $\delta_{t}$ are families of functions, $g_{t}(0)=\delta_{t}(0)=0$. The solvability of this equation follows from its solvability in formal series with respect to $z$ and hyperbolicity of the vector field $z(\partial / \partial z)+z E_{t}$ on the hypersurface $\{z=0\}$ (see [Zh, 92], Ch. 2 and [RZh, 95]). It remains to note that $v_{t}(0)=0$ since $\delta_{t}(0)=0$.

c) Let $(\omega, f) \in \operatorname{Orb}\left(Q, x_{1}\right) \cup \operatorname{Orb}\left(Q, x_{2}+x_{1}^{2}\right)$. Then $\omega \wedge(d \omega)^{k-1}(0) \neq 0$ and by Darboux theorem the field of kernels of $\omega$ is equivalent to the field of kernels of the 1-form $Q$. Therefore there is no loss of generality to assume that $\omega=Q$. If $(Q, f) \in \operatorname{Orb}\left(Q, x_{1}\right)$ then $\left(Q \wedge(d Q)^{k-1} \wedge d f\right)(0) \neq 0$, or, equivalently, $\left(\partial f / \partial x_{1}\right)(0) \neq 0$. The latter condition implies that $(Q, f)$ is equivalent to $\left(Q, x_{1}\right)$.

Assume now that $(Q, f) \in \operatorname{Orb}\left(Q, x_{2}+x_{1}^{2}\right)$. Then the function $f$ has the following properties:

$$
\frac{\partial f}{\partial x_{1}}(0)=0, \frac{\partial^{2} f}{\partial x_{1}^{2}}(0) \neq 0, \quad(Q \wedge d f)(0) \neq 0 .
$$

It follows that $(Q, f)$ is equivalent to $\left(Q, \pm x_{1}^{2}+\nu\right)$, where $\nu$ is a function of the variables $y_{1}, x_{2}, y_{2}, \ldots, x_{k}, y_{k}$ such that $(Q \wedge d \nu)(0) \neq 0$. By a) the affine distribution $(Q, \nu)$ on $R^{2 k-1}\left(y_{1}, x_{2}, y_{2}, \ldots, x_{k}, y_{k}\right)$ is equivalent to $\left(Q, x_{2}\right)$. Then $\left(Q, \pm x_{1}^{2}+\nu\right)$ is equivalent to $\left(Q, x_{2} \pm x_{1}^{2} T\right)$, where $T$ is a nonvanishing function. Changing the coordinate $x_{1}$ we can 
reduce $T$ to 1 . It remains to note that $\left(Q, x_{2}-x_{1}^{2}\right)$ is equivalent to $\left(Q, x_{2}+x_{1}^{2}\right)$ since the change of the signs of the coordinates $y_{1}, x_{2}, \ldots, x_{k}$ brings $Q$ to $-Q$.

3.3. Proof of Theorem 2. Throughout the proof we use Theorem 3 and the description of the orbits in Sections 1.6 and 2.2. At first we consider the odd-dimensional case, $n=2 k+1$. Denote by $S_{1}$ the union of the orbits of the models $(D, 1)$ and $\left(M_{ \pm}, 1\right)$ and by $S$ the union of the orbits of the models $(D, 1),\left(M_{ \pm}, 1\right),\left(D, x_{1}\right)$ and $\left(M_{ \pm}, x_{1}\right)$. Given a 1-form $\omega$ we associate to it a function $F=\omega \wedge(d \omega)^{k} / \Omega$, where $\Omega$ is a nondegenerate volume form. Introduce the following sets of germs of corank one affine distributions:

$$
\begin{aligned}
B_{1}=\{(\omega, f): \quad F(0) & \left.=0,\left(f(d \omega)^{k} \wedge d F+k \omega \wedge(d \omega)^{k-1} \wedge d f \wedge d F\right)(0)=0\right\} ; \\
B_{2} & =\{(\omega, f): f(0)=0,(\omega \wedge d f)(0)=0\} .
\end{aligned}
$$

Lemma 1. Let $f(0) \neq 0$. Then $(\omega, f) \in S_{1}$ if and only if $(\omega, f) \notin B_{1}$.

To prove the lemma we write the affine distribution $(\omega, f)$ in the form $(\omega / f, 1)$ and use the description of the models in Section 1.6.

Using the description of the models in Section 2.2 we obtain the following corollary: $(\omega, f) \in S$ if and only if $(\omega, f) \notin B_{1} \cup B_{2}$. It follows that the set of 2-jets of germs of $S$ is open and we obtain that any germ of $S$ is simple. Another corollary of Lemma 1 (and results of Section 1.6) is the absence of simple germs in the set $B_{1} \cap\{(\omega, f): f(0) \neq 0\}$ and thus in the set $B_{1}$.

To prove Theorem 2 in the odd-dimensional case it remains to show that there are no simple germs in the set $B_{2}$. It suffices to prove that a germ $(\omega, f) \in B_{2}$ such that $d f(0) \neq 0$ is not simple. Let $\mu=\mu(\omega, f)$ be the pullback of $\omega$ to the hypersurface $\{f=0\}$. Note that $\mu(0)=0$ and that if $(\omega, f)$ is equivalent to $(\tilde{\omega}, \tilde{f})$ then the Pfaffian equations generated by $\mu(\omega, f)$ and $\mu(\tilde{\omega}, \tilde{f})$ are equivalent. It follows that the problem of classification of generic germs of the set $B_{2}$ contains the problem of classification of generic Pfaffian equations generated by 1 -forms which vanish at 0 . In the latter problem there are no simple germs, see [L, 75] and [Zh, 92], Sect. 21, 29.

Now we consider the even-dimensional case, $n=2 k \geq 4$. At first let us note that a germ $(\omega, f)$ such that $\left(\omega \wedge(d \omega)^{k-1}\right)(0)=0$ is not simple since in this case the germ of the Pfaffian equation generated by $\omega$ is not simple, see [Zh, 92], Sect. 25. Therefore it suffices to prove Theorem 2 within the set $C$ consisting of germs of the form $(Q, f)$. Denote by $C_{1}$ the subset of $C$ consisting of germs $(Q, f)$ such that $\left(\partial f / \partial x_{1}\right)(0)=\left(\partial^{2} f / \partial x_{1}^{2}\right)(0)=0$ and by $C_{2}$ the subset of $C$ consisting of germs $(Q, f)$ such that $f(0)=0,(Q \wedge d f)(0)=0$. Using the description of orbits in Sections 1.6 and 2.2 it is easy to show that a germ $(Q, f)$ belongs to the union $S^{\prime}$ of the orbits of the models $\left(D^{\prime}, 1\right),\left(M_{ \pm}^{\prime}, 1\right),\left(Q, x_{1}\right),\left(Q, x_{2}+x_{1}^{2}\right)$ if and only if $(Q, f) \notin\left(C_{1} \cup C_{2}\right)$. This observation and results of Section 1.6 imply that (i) the set of 2-jets of germs of the set $S^{\prime}$ is open, therefore any germ of $S^{\prime}$ is simple; (ii) there are no simple germs belonging to the set $C_{1} \cap\{(\omega, f): f(0) \neq 0\}$, therefore there are no simple germs in the set $C_{1}$. Arguing as in the odd-dimensional case we obtain that there are no simple germs $(Q, f) \in C_{2}$, therefore any simple germ belongs to $S^{\prime}$. 


\section{References}

[AVG, 85] V. I. Arnol' d, S. M. Guseŭn-Zade, A. N. Varchenko, Singularities of Differentiable Maps, vol. 1, Birkhäuser, Boston, 1985.

[Ar, 78] V. I. Arnol'd, Mathematical Methods in Classical Mechanics, Springer-Verlag, New York, 1978.

[J, 90] B. Jakubczyk, Equivalence and invariants of nonlinear control systems, in: Nonlinear Controllability and Optimal Control, H. Sussmann (ed.), Marcel Dekker, New York, 1990, 177-218.

[JR, 90] B. Jakubczyk, W. Respondek, Feedback equivalence of planar systems and stabilizability, in: Robust Control of Linear Systems and Nonlinear Control, Proc. of the Internat. Sympos. MTNS-89, vol. 2, M. A. Kaashhoek, J. H. van Schuppen, and A. C. M. Ran (eds.), Birkhäuser, Boston, 1990, 447-456.

$[\mathrm{L}, 75] \quad$ V. Lychagin, Local classification of nonlinear first-order partial differential equations, Uspekhi Mat. Nauk 30 (1975), 101-171; English transl. Russian Math. Surveys 30 (1975).

[M, 70] J. Martinet, Sur les singularites des formes differentielles, Ann. Inst. Fourier (Grenoble) 20 (1970), 95-178.

[RZh, 95] W. Respondek, M. Zhitomirskii, Feedback classification of nonlinear control systems on 3-manifolds, Math. Control Signals Systems 8 (1995), 299-333.

[T, 89] K. Tchoń, On normal forms of affine systems under feedback, in: New Trends in Nonlinear Control Theory, J. Descusse, M. Fliess, A. Isidori, and D. Leborgne (eds.), Lecture Notes in Control and Information Sciences 122, Springer-Verlag, 1989, 23-32.

[Zh, 85] M. Zhitomirskii, Finitely determined 1 -forms $\omega,\left.\omega\right|_{0} \neq 0$ are exhausted by the models of Darboux and Martinet, Funktsional Anal. i Prilozhen. 19 (1985), 71-72; English transl. Funct. Anal. Appl. 19 (1985).

[Zh, 92] M. Zhitomirskii, Typical Singularities of Differential 1-Forms and Pfaffian Equations, Translations of Mathematical Monographs 113, AMS, Providence, 1992. 